\title{
Non- Isolation Treatment of Atrial Fibrillation: Does Autonomic Nerve Modulation Really Act?
}

\author{
Hong-Tao Wang et al.,* \\ Tangdu Hospital, Fourth Military Medical University, Xi' an,
}

China

\section{Introduction}

Atrial fibrillation (AF) is an abnormal heart rhythm that originates in the top chambers of the heart (atria). Pulmonary vein (PV) ablation is an effective treatment for AF, which has been used widely in clinic; however, significant side effects are difficult to avoid including potential recurrence, pulmonary vein stenosis, systemic thromboembolism, pericardial effusion, cardiac tamponade, esophageal perforation, and phrenic nerve paralysis.

These limitations suggest that PV isolation is not a majority determinant of clinical success and promote research toward the development of less aggressive, but equally effective, procedures. First, researchers focus on some non-anti-arrhythmic drug therapy. There is accumulating evidence in support of the anti-arrhythmic effects of non-anti-arrhythmic drugs. Treatments with angiotensin-converting enzyme inhibitors, angiotensin-receptor blockers, statins, and omega-3 fatty acids all seem promising, over and above any effect related to the treatment of underlying heart disease. Despite exciting results from animal experiments and promising outcomes from retrospective analyses, there is no robust evidence of specific effects of these drugs to transform current clinical practice (Ardell et al., 2001). Therefore, autonomic nerve modulation has recently become a new strategy for AF management.

This chapter aims to study the relationship between ANS and AF; and to investigate the effect of autonomic nerve modulation on AF.

\section{The relationship between ANS and the atrial fibrillation}

\subsection{Innervation of ANS to the heart}

The cardiac ANS contains two main components: the extrinsic and intrinsic ANS. The extrinsic ANS is defined as the nerves coming from the brain and thoracic paravertebral ganglia, consisting mainly of ganglia and their axons located outside the heart.

The extrinsic ANS is composed of the sympathetic system and the parasympathetic system. The cardiac sympathetic ganglia include a superior cervical ganglion, which communicates with C1-3, and the cervicothoracic (stellate) ganglion, which communicates with C7-8-T1-2. In addition, the thoracic ganglia (as low as the seventh thoracic ganglion) contribute to sympathetic innervation of the heart. The superior, middle, and inferior cardiac nerves from

\footnotetext{
* Qiang-Sun Zheng, Xiong-Tao Liu, Jun Li, Fu-Jun Shang, Fei-Fei Su, Bo-yuan Fan and Dong-Bo Ou The first 3 authors contributed equally to this work.
} 
these ganglia innervate the heart by following a simple course along the brachiocephalic trunk, common carotid and subclavian arteries. On the other hand, the thoracic cardiac nerves in the posterior mediastinum must follow a complex course to reach the heart in the middle mediastinum. Parasympathetic innervation comes from the vagus and is divided into superior, middle, and inferior branches. Although both sides of the autonomic branches run through the ventral and dorsal aspects of the aortic arch, the right autonomic cardiac nerves tend to follow a ventral course. The main vagal trunk then continues along the esophagus to reach the gut.

The intrinsic ANS is composed mainly of ganglionated plexi (GP) and their axons situated on the heart itself or along the great vessels in the thorax (Scherlag et al., 2006). These GPs usually are embedded in the fat pads located on the epicardium. Prior studies showed multiple GPs located on the atria of mammalian hearts, including the anterior right GP located at the caudal end of the sinoatrial (SA) node, the inferior right GP situated at the junction of inferior vein cava and both atria, and the superior left GP located adjacent to the base of the left superior pulmonary vein between the left atrial appendage and the left pulmonary artery (another appellation is right pulmonary artery ganglionated plexi; RPA GP, because this GP is also adjacent to the right pulmonary artery). The anterior right GP is also referred to as the right superior pulmonary vein fat pad or SA fat pad because of its close proximity to the right superior pulmonary vein and SA node; the inferior right GP is sometimes called the AV node fat pad because of its role in modulating the AV conduction; and the superior left GP is often referred to as the left superior pulmonary vein fat pad because of its close proximity to the left superior pulmonary vein (Hou, et al., 2007). However, the specific intrinsic autonomic efferent pathway to the atrium and PVs remains unknown when reaches to the region of superior vena cava (SVC). This area is adjacent to the aortic root, contains the SLGP, the surrounding fat tissues and some dispersed GPs.

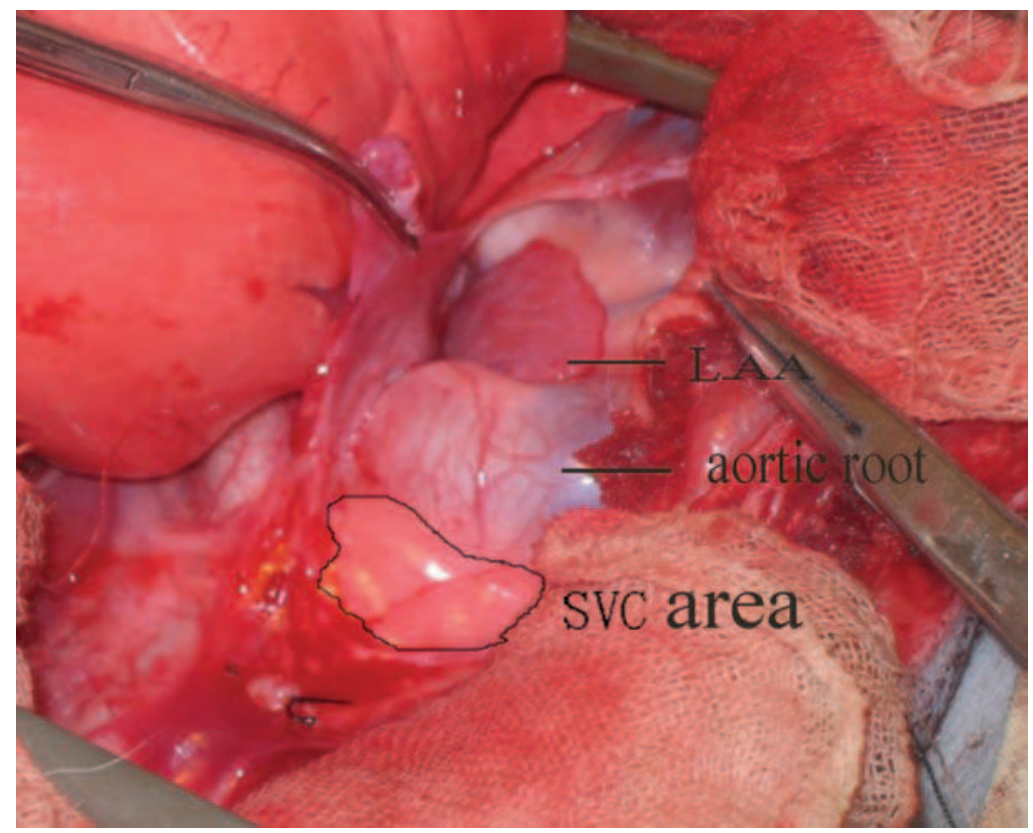

Fig. 1. Location of the SVC area. 
Illustration: The SVC area is adjacent to the aortic root, contains superior vena cava and aortic root ganglionated plexi, surrounding fat tissues and some dispersed GPs.

\section{The function of the SVC area}

\subsection{Identifying the neuron cell type of the SVC area}

Hearts were excised from the mongrel dogs, and a cannula was infused into the aorta ascendent. This procedure was followed by perfusion with $250 \mathrm{ml}$ physiologic saline to clean the hearts. The SVC area was isolated from the aortic root, perfused and fixed with $1 \mathrm{~L}$ $4 \%$ formalin, and stored in 30\% sucrose overnight after an additional post-fixation for $4 \mathrm{~h}$ in $4 \%$ formalin. The tissues were cut into three parts and embedded in OCT. Tissues were cut into 20 -mm sections at $-25^{\circ} \mathrm{C}$ with a cryostat.

The SVC area was cut into 3 parts, and Nissl's staining was used to determine the location of the GPs. The results reveal that neurons mix with fat cells, are widely distributed in the GPs, and are present in small clusters of 3 to 15 cells with most embedded in the right part of SVC area (Figure 2).
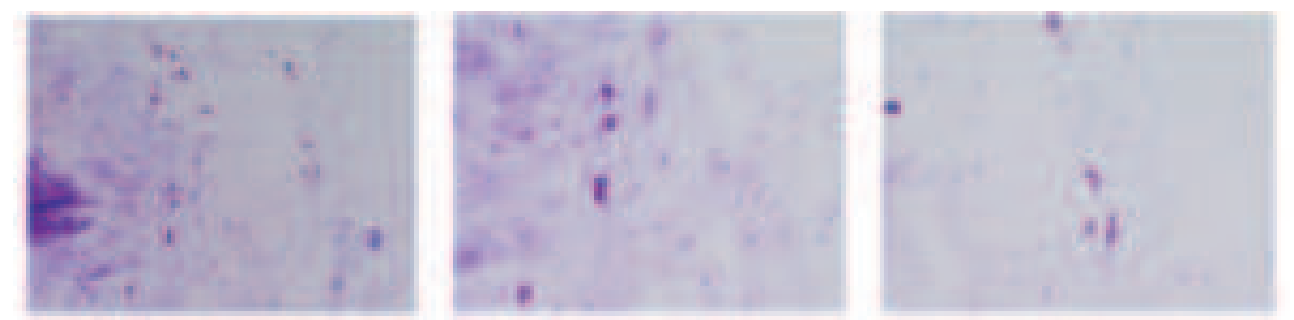

Fig. 2. Location of the ganglionated plexuses in SVC area.

Illustration: Nissl's staining was performed to reveal the distribution of ganglionated plexuses (GPs). Figure 2A ( $1 \times 20$ eye lens) shows that GPs are widely distributed in the SVC area and are surrounded by many fat cells. Figures $2 B$ and $2 \mathrm{C}(1 \times 40$ eye lens) show that GPs are present in small clusters of 7 and 5 cells, respectively.

The study by Chiou et al (Chiou et al., 1997) showed that the SVC FP receives most vagal nerves to both atriums. Chiou et al. claimed that stimulation of this FP shortened ERP of atriums, prolonged spontaneous cycle length, and induced AV block. Catheter ablation of this FP could vagally denervate the atrium. This GP located in the right side of the aortic root, and did not include the surrounding fat tissues. Therefore, the right part was more likely to be the location of SVC-Ao FP. There were also GPs in the middle and left part of the aortic root. These GPs and the surrounding fat tissues were also important components of the SVC area that could not be ignored.

Fluorescent dual-labeling with antityrosine hydroxylase $(\mathrm{TH})$ and anticholine acetyltransferase (ChAT) antibodies was performed to determine the neuron cell types in the GPs and to determine whether both adrenergic and cholinergic nerves co-exist within the GPs. Fluorescein-labeled anti-mouse IgG was used as the secondary antibody. Fluorescein isothiocyanate (FITC) was conjugated to TH, and Rhodamine-Labeled AntiGoat IgG was conjugated to ChAT. The dual-labeled sections were then visualized with a laser-scanning confocal microscope.

Fluorescent double staining showed complex categories of neurons in the SVC area: adrenergic and cholinergic neurons were both observed, but the majority of neurons in the 
GP were of the cholinergic subtype (Figure 3A, 3B). Moreover, a proportion of neurons expressed dual adreno-cholinergic phenotypes (Figure 3C).
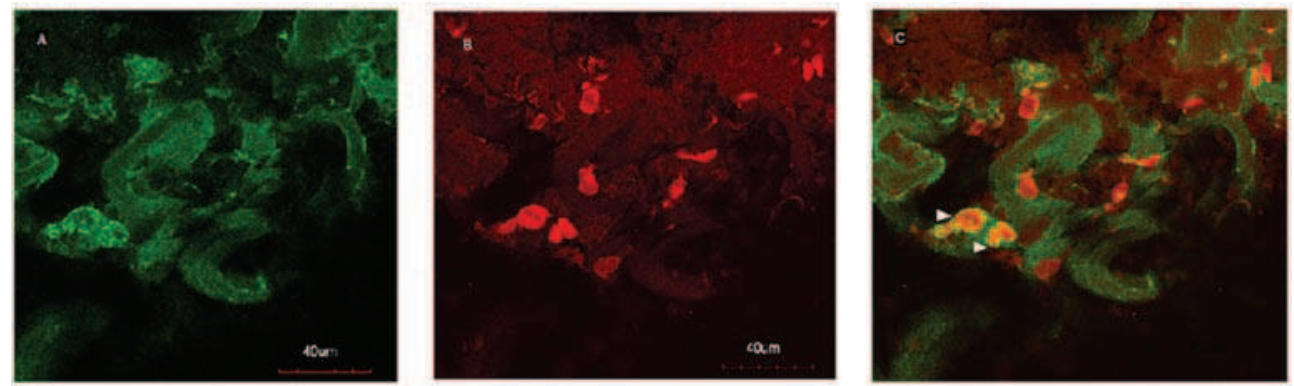

Fig. 3. Neuronal cell types in the ganglionated plexuses of SVC area.

Illustration: Fluorescence photomicrographs show that $\mathrm{TH}$ and ChAT are co-expressed in GPs of the SVC area. Figure 3A shows the TH (green)-labeled cholinergic neurons; Figure 3B shows the ChAT (red)-labeled adrenergic neurons; and Figure 3C shows the co-expression of TH and ChAT (white arrow).

These results suggest that ganglion cells can potentially simultaneously express both cholinergic and adrenergic markers, which may occur in the process of a neurochemical switch in response to external stimuli. These cells have also been observed in human pulmonary vein-atrial junction (Tan et al., 2006). This result indicates that stimulation/excision of GPs actually influenced the whole ANS, neither the vagal nor the sympathetic system alone, and the vagal effect was predominant because cholinergic neurons were the majority.

\subsection{Efferent autonomic pathway to the atrium and PVs}

Five microliters of $10 \%(\mathrm{w} / \mathrm{v})$ biotinylated dextran amine (BDA) anterograde tracer (MW 10,000; lysine fixable; Molecular Probes, Invitrogen) dissolved in 0.05 M PBS was injected into the SVC area with micropipettes. A total of 5 injection sites - being adjacent to the root of the right part of the SVC area - were selected, and the micropipettes were used at each injection site for $5 \mathrm{~min}$. The dogs were sacrificed 3 days after BDA injection.

Both the atria and the four pulmonary veins were cut into $10-\mu \mathrm{m}$-thick sections on a cryostat. The series of sections were then processed for BDA, TH, and ChAT triple immunofluorescence histochemistry. Avidin-conjugated CY5 was used to label BDA, and both $\mathrm{TH}$ and ChAT were labeled as described above. Finally, the labeled slides were examined under a laser-scanning confocal microscope.

Injection of an anterograde tracer into the SVC area showed that BDA-containing varicose fibers (Figure 4, A) projected directly into the LA and LPV, while TH-labeled (Figure 4, B) and ChAT labeled (Figure 4, C) axons and varicosities were observed within the same traced varicose fibers. A total of $101 \mathrm{BDA}$-positive varicose fibers were counted in the LA and LPVs of five animals, and the counts were obtained for 20 sections (four sections in each animal). Of the fibers, 23 (22.7\%) were only ChAT-immunoreactive, and 20 (19.8\%) displayed both $\mathrm{TH}$ and ChAT-like immunoreactivity. Double-immunostaining of $\mathrm{TH}$ and ChAT in the absence of BDA was also observed in both the LA and LPV (Figure 4, D). 

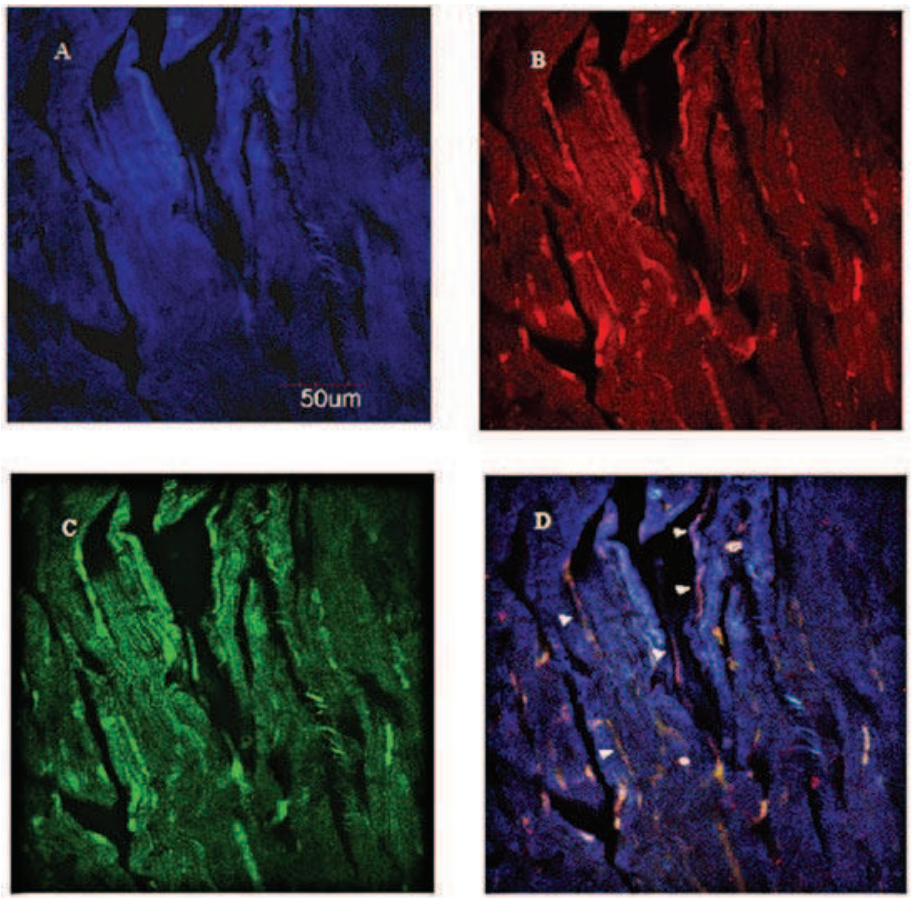

Fig. 4. Co-expression of TH and ChAT in the left atrium and left pulmonary veins.

Fluorescence photomicrographs showed BDA-labeled autonomic fibers projected from the SVC AREA to both the LA and LPV. BDA, ChAT, and TH immunoreactivities were visualized with Avidin-conjugated CY5 (A, blue), Rhodamine (B, red), and FITC (C green, antibodies, respectively. The merged digital image (D) showed triple staining of ChAT, TH, and BDA (white arrow).

In contrast to our initial predictions, BDA-positive varicose fibers were not found in the RA or RPV. It is possible that the nerve fibers travel to other GPs (i.e., SLGP, ARGP, or IRGP) first and then project into the RA and RPV.

From the above study, we demonstrated parasympathetic and sympathetic neural elements were highly co-localized in the SVC area and mixed with fat cells. We could not separate cholinergic neurons from adrenergic ones or neuron cells from fat cells. This result explains why the whole SVC area, including the fat cells, needs to be all excised to eliminate the intrinsic ANS effect.

\section{The effect of ANS modulation on atrial fibrillation}

\subsection{Review of ANS modulation}

Autonomic GPs play an important role in the initiation and maintenance of AF. Several observations suggest that the ANS is crucial in both the initiation and/or the maintenance of $\mathrm{AF}$ in humans. Changes in autonomic tone, especially the vagal tone, may be involved in the generation of ectopic PV activity (Schauerte et al., 2001; Sharifov et al., 2004). Vagal nerve stimulation shortens AERP, decreases the wavelength of atrial reentrant circuits and 
increases dAERP, thus, promoting the stability of AF (Li et al., 2007). However, SLGP acts as the "head station" of vagal inputs to both atria and the sinus and AV nodes in the dog. Most patients with idiopathic PAF appear to be vagally dependent, with heightened susceptibility to vasovagal cardiovascular response. Studies have demonstrated that modification of GPs affected atrial vagal inputs and prevented AF initiation (Lemola et al., 2008). In contrast, in most patients with organic heart diseases, the PAF episodes appear more sympathetically dependent (Huang J.L. et al., 1998). A shift toward an increase in sympathetic tone or a loss of vagal tone has been observed before postoperative PAF, before the onset of atrial flutter, and before PAF occurring during sleep, whereas a shift toward vagal predominance was observed in young patients with lone AF and nocturnal episodes of PAF. More recently, it has been observed that a primary increase in adrenergic drive followed by marked modulation toward vagal predominance immediately before the onset of PAF (Chen P.S. et al., 2007).

Atrial autonomic nerve density is highest in the region of the PVs. One area rich in autonomic innervation is the PV-LA junction which may contribute to the initiation of AF (Chen et al., 2007). Recently, some progresses have been made in ANS modulation. Support for the autonomic basis of paroxysmal and persistent forms of atrial fibrillation have been forthcoming from several clinical series. In 2004, Platt et al. (Platt M et al., 2004) reported that 22 of 23 patients (96\%) with persistent atrial fibrillation who had ganglionated plexi ablation alone (no pulmonary vein isolation) were non-inducible immediately after the procedure and at a short follow-up period (median 6 months). Importantly, the number of radiofrequency applications delivered ranged from 5 to 17 (Pappone $C$ et al., 2004) reported that during the application of radiofrequency to induce linear lesions to encircle the pulmonary veins, a 'vagal' response was noted in 100 patients in a series of 297 consecutive cases. This took the form of slowing of the sinus rate during sinus rhythm or a slowing of the ventricular response during atrial fibrillation. While continuing the radiofrequency application, these responses disappeared. A 12-month follow-up showed that the $34.4 \%$ who appeared the vagal response had a $99 \%$ freedom from atrial fibrillation recurrence whereas the other group had an $85 \%$ success rate, that is, freedom from recurrence. Scherlag (Scherlag et al., 2006) stated that ablation of only the ganglionated plexi in the cadiac fat pads may result in higher success rates and may reduce damage incurred to healthy myocardium.Tan (Tan et al., 2008) further demonstrated that cryoablation of extrinsic sympatho-vagal nerves eliminated paroxysmal atrial fibrillation.

These results indicate that rapid ANS modulation does act in the treatment of AF. However, the effect and mechanism of ANS modulation on AF originating in PVs is still unclear. We all know that facal repetitive activities arise from PVs, conduct to the left atria through P-A junction and initiate AF. The following study will investigate Whether ANS modulation can decrease these facal activities in PVs and prevent AF occurrence?

\subsection{SLGP stimulation and ablation}

To stimulate the SLGP in this FP, a self-made electrode with eight metal electrode heads was used.

The radiofrequency energy was delivered to the SLGP through a 7F deflectable quadripolar catheter with a 4-mm-tip electrode. The catheter tips were placed manually on the surface of the SLGP and the surrounding fat tissues under direct visualization to ensure ideal tissue contact and energy delivery. Continuous unmodulated radiofrequency current (300 to 750 
$\mathrm{kHz}$ ) with a power output setting of 30 to $35 \mathrm{~W}$ was delivered from a radiofrequency generator for a duration of 60 seconds.

The results (Wang HT et al., 2010) showed that SLGP stimulation changed atrial electrophysiological properties. Compared with the baseline group, stimulation shortened AERP, LSPVERP, LIPVERP, RSPVERP, and RIPVERP while it increased dAERP .

Catheter ablation reversed the changes in electrophysiological properties caused by SLGP stimulation. Compared with the baseline group, ablation prolonged AERP, LSPVERP, LIPVERP, RSPVERP, RIPVERP, and decreased dAERP (Figure 5).

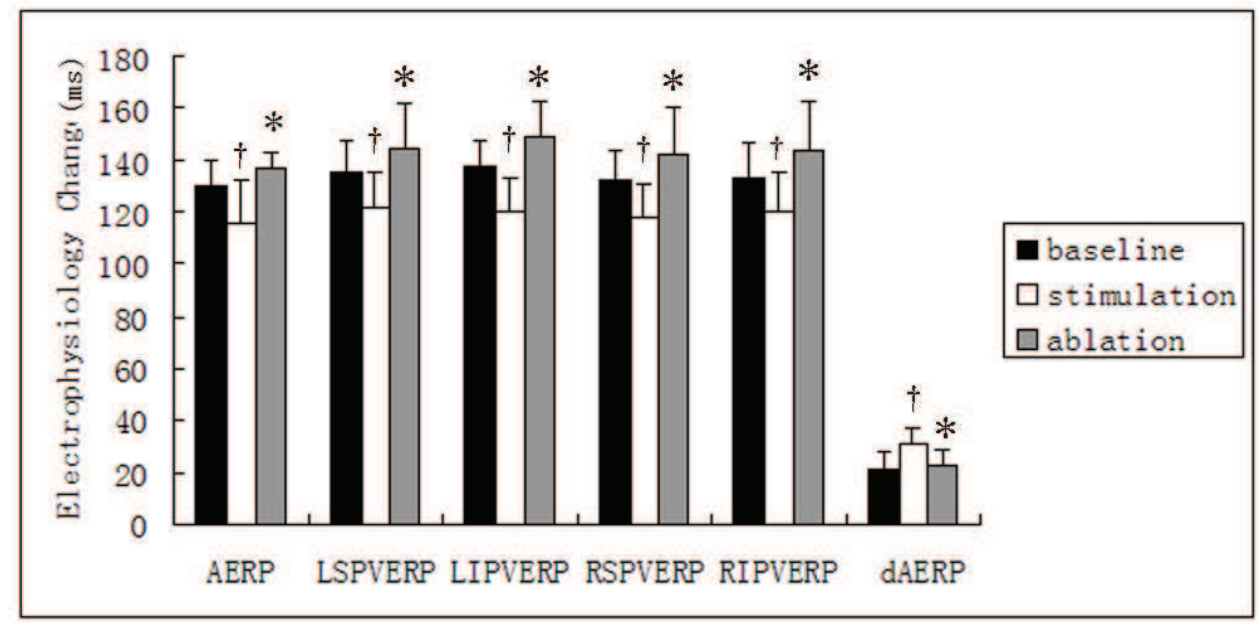

Fig. 5. Electrophysiological changes during RPA FP/GP modification.

Illustration: Changes of AERP, dAERP, PVERP at baseline, during RPA FP/GP stimulation or after ablation. RPA FP/GP stimulation significantly shortened AERP and PVERP and increased dAERP at all atrial sites compared with baseline $(\mathrm{P}<0.01)$. After ablation, AERP and PVERP increased and dAERP decreased significantly compared with baseline $(\mathrm{P}<0.05)$. ${ }^{*} \mathrm{P}<0.05$ vs., baseline; $\uparrow \mathrm{P}<0.01$ vs., baseline.

\subsection{Induction of AF}

$\mathrm{AF}$ was initiated from the distal part of the four PVs. The recording electrode was placed in the proximal PV, and the distance between the stimulating electrode and the recording electrode was about $2 \mathrm{~cm}$. AF was first provoked by programmed electrical stimulation (S1S2). If S1S2 failed to induce arrhythmia, burst pacing (S1S1) at a cycle length of $200 \mathrm{~ms}$ for 30 seconds was applied to provoke AF in the remaining dogs, and the cycle length was decreased to 160 and $120 \mathrm{~ms}$ in turn if $200 \mathrm{~ms}$ was ineffectual. The percent of AF induced was measured to evaluate AF inducibility.

The results (Wang HT et al., 2010) showed that AF was induced in 11 of 20 dogs from distal LSPV (Table 1) at baseline without VS stimulation. S1S2 provoked 3 AF episodes, and S1S1 provoked the other 8 (Table 1). vagosympathetic stimulation (VS) significantly increased AF episodes to 18. S1S2 provoked 11 AF episodes, and S1S1 provoked the other 7 (Table 1). After ablation during VS stimulation, AF was no longer inducible by S1S2 in any dogs. S1S1 could still induce AF in 8 of 20 dogs, but the percent induced decreased to $40 \%$ (Table 1). 
The same protocol was performed at distal LIPV, RSPV, and RIPV at baseline without VS stimulation, and the percent of $\mathrm{AF}$ induced was $45 \%, 50 \%$, and $50 \%$, respectively (Table 1 ). In contrast, at baseline with VS stimulation, the percent induced increased to $80 \%, 75 \%$, and $70 \%$, respectively (Table 1). However, after ablation during VS stimulation, AF episodes decreased to $30 \%, 30 \%$, and $25 \%$, respectively, compared with baseline values (Table 1), and these episodes were all induced by S1S1.

\begin{tabular}{|c|c|c|c|c|c|}
\hline & & S1S2 & S1S1 & Total & $(\%) n=20$ \\
\hline \multicolumn{6}{|l|}{ LSPV } \\
\hline Baseline & VS- & 3 & 8 & 11 & 55 \\
\hline & VS+ & 11 & 7 & 18 & $90^{*}$ \\
\hline Ablation & & 0 & 8 & 8 & 40 \\
\hline LIPV & & & & & \\
\hline Baseline & VS- & 2 & 7 & 9 & 45 \\
\hline & VS+ & 8 & 8 & 16 & 80 \\
\hline Ablation & & 0 & 6 & 6 & 30 \\
\hline RSPV & & & & & \\
\hline Baseline & VS- & 2 & 8 & 10 & 50 \\
\hline & VS+ & 7 & 8 & 15 & 75 \\
\hline Ablation & & 0 & 6 & 6 & 30 \\
\hline $\begin{array}{l}\text { RIPV } \\
\text { Baseline }\end{array}$ & VS- & 1 & 9 & 10 & 50 \\
\hline & VS+ & 7 & 7 & 14 & 70 \\
\hline Ablation & & 0 & 5 & 5 & 25 \\
\hline
\end{tabular}

Table 1. Comparison of AF incidence before and after RPA FP/GP ablation.

$\%$ refers to the percent induced of AF. VS + indicates baseline with vagosympathetic stimulation and VS - indicates baseline without vagosympathetic stimulation. Ablation refers to vagosympathetic stimulation post ablation of $\mathrm{GP} * \mathrm{P}<0.01$ vs. baseline.

Programmed stimulation (S1S2) and burst stimulation (S1S1) were performed in turn to provoke AF from distal PVs. In 20 dogs, the percent of AF induced decreased significantly after RPA FP/GP ablation compared with baseline groups.

In conclusion, AF was less likely to be induced from PVs after SLGP ablation. Although the mechanism is unclear, the results may be relevant not only for the independent modulation of the intrinsic autonomic system, but also for the improvement of electrophysiological properties after ablation-less dAERP and longer AERP and PVERP, which decreased the number of atrial reentrant circuits and made re-entry more difficult. Recently, studies showed that ablating only SLGP or SLGP plus IVC-LA GP partially denervates atrial autonomic nerves and increases the incidence of AF (Hirose et al., 2002; Oh et al., 2006). Partial denervation of the atrium was therefore avoided in AF treatment. However, our findings demonstrate that partial denervation of atrial autonomic nerves by ablating SLGP decreased inducibility of AF originating in PVs. This finding suggests that the three major FPs play different roles in AF initiation, and partial denervation is not always harmful to control AF. 


\section{Prospect-the role of the parasympathetic signal pathway in AF}

Except the above mass electrophysiology and histological study, we also studied muscarinic type- 2 receptors (M2R) and inward rectifying $\mathrm{K}+$ current (IKACh), which were the key roles of parasympathetic signal pathway.

The M2 muscarinic receptors reside in both the atria and ventricles but have a greater density in the former. The known effects of the parasympathetic nervous system on cardiac function, heart rate, and atrioventricular nodal conduction appear modulated generally via M2 R. The M2R plays an important role in AF. The densities of $M 2 R$ was spatial heterogeneity combined with the heterogeneous distribution of parasympathetic innervations of the atria, which may contribute to the proarrhythmic ability of vagal nerve stimulation. Cong-Xin Huang (Huang CX et al., 2006) found the density densities of M2R in LAA, RAA and LA were higher than that in RA, PV and SVC. Furthermore, we demonstrated (Hong et al., 2009) that autoantibodies against M2R (M2-AAB) can induce atrial electrophysiology remodeling, atrial fibrosis and upregulation of the M2R and IKACh. These results suggest that the presence of M2-AAB may participate in the induction and perpetuation of AF. And we also demonstrated (Yang et al., 2009) that the distribution of atrial M2R was heterogeneous and had the greatest density in the left atrial free wall (LAFW).The epicardial side had greater M2R density than the endocardial side. We found the distribution of atrial M2R changed with age. Increased density of the M2R in the LAFW accompanied senescence. This variation may enhance the heterogeneity of the M2R distribuion and may contribute to age-related changes in AF vulnerability.

The IKACh is present in the sinoatrial node, atria, atrioventricular node, and Purkinje fibers of the mammalian heart and coupled to muscarinic type- 2 receptor and adenosine type- 1 receptors through pertussis toxin (PT) or select-activating protein -sensitive G-proteins (GK). The IKACh possesses three distinct open/closed states. IKACh is comprised of two homologous proteins: GIRK1 and GIRK4. In the heart, only the expression of Kir3.1 and Kir3.4 mRNAs is detected. GIRK4 is the functional subunit of IKACh and ablation of GIRK4 typically results in the functional elimination of IKACh. Moreover, GIRK1 cannot form a functional ion channel in the absence of GIRK4.

The cardiac acetylcholine-sensitive $\mathrm{K}+$ channel plays a major role in the parasympathetic regulation of heart rate, and is also crucial in the generation and maintenance of AF. First, IKACh was spatial heterogeneity in the atria which facilitated the generation and maintenance of AF. Cong-Xin Huang (Huang CX et al., 2006) found the densities of IKACh in LAA, RAA and LA were higher than that in RA, PV and SVC in LAA, RAA. Sarmast F (Sarmast F et al., 2003) found a greater abundance of Kir3.x channels and higher IKACh density in LA than RA myocytes result in greater ACh-induced speeding-up of rotors in the LA than in the RA. A canine form of constitutively active IKACh(called IKH) shows regional heterogeneity (larger in PV cardiomyocyte sleeves).Secondly, the IKACh participated the electric remodeling of atrial fibrillation. Kir3.1 mRNA levels are downregulated in human AF. In contrast to reduced functional IKACh and Kir3 subunit mRNA content, an increase in a constitutively active form of this current has been described as a potentially important contributor to the AF substrate.Furthermore, IKACh is a potentially interesting target for atrial-selective anti-AF therapy. The suggested activation of IKACh predisposed to AF and lack of IKACh prevented AF. But it is still unknown the 
precise mechanism of IKACh inhibition to the antiarrhythmic potency of these drugs and selective inhibitors are not yet available for clinical application. We successfully used (Liu et al.,2009) adenovirus-delivered short hairpin RNA targeting GIRK4 not only decreased the expression of GIRK4 in human atrial myocytes, but also affected IKACh densities. If we attempted to acquire more effective and persistent RNAi effects in human atrial myocytes and use adenovirus-delivered small hairpin RNA against GIRK4 in clinic, there are a great deal challenges to be overcome.

Although the role of the parasympathetic signal pathway is accpepted both in initiation and maintenance of AF. It is less clear in AF patients whether inhibition of parasympathetic activity have an anti-arrhythmic effect. Furthermore, persistent and selective inhibition was still difficult.

In conclusion, ANS modulation plays an important role in the treatment of AF. The mechanism remains unclear. And the change of ion-channel current should be investigated to disclose the mechanism of ANS modulation in the control of AF. Maybe more penetrating studies are needed to associate ANS modulation with ion-channel inhibition to probe a new way to improve the treatment of AF.

\section{References}

Armour J.A., \& Ardell J.L. (1994). Neurocardiology (Second edition.). Structure and function of mammalian intrinsic cardiac neurons. Oxford University Press, New York, pp, 95-114.

Chen P.S., \& Tan AY. (2007). Autonomic nerve activity and atrial fibrillation. Heart Rhythm, 4, S61- S64.

Chiou C.W., Eble J.N., \& Zipes D.P. (1997). Efferent vagal innervation of the canine atria and sinus and atrioventricular nodes. Circulation, 95, 2573-2584.

Hirose M, Leatmanoratn Z, Laurita K.R., \& Carlson M.D. (2002). Partial vagal denervation increases vulnerability to vagally induced atrial fibrillation. J Cardiovasc Electrophysiol,13, 1272-1279.

Hong C.M., Zheng Q.S., Liu X.T., Shang F.J., Wang H.T., \& Jiang W.R. (2009). Effects of Autoantibodies against M2 Muscarinic Acetylcholine Receptors on Rabbit Atria in vivo. Cardiology, 112,180-187.

Hou Y, Scherlag B.J., Lin J, Zhou J, Song J, Zhang Y, Patterson E, Lazzara R, Jackman W.M., \& Po S.S. (2007). Interactive atrial neural network: Determining the connections between ganglionated plexi. Heart Rhythm, 4, 56-63.

Huang C.X., Zhao Q.Y., Liang J.J., Chen H, Yang B, Jiang H, \& Li G.S. (2006). Differential densities of muscarinic acetylcholine receptor and $\mathrm{I}_{\mathrm{KACh}}$ in canine supraventricular tissues and the effect of amiodarone on cholinergic atrial fibrillation and I KACh. Cardiology, 106, 36-43.

Huang J.L., Wen Z.C., Lee W.L., Chang M.S. \& Chen S.A.(1998).Changes of autonomic tone before the onset of paroxysmal atrial fibrillation, Int J Cardiol, 66 , 275-283.

Lemola K, Bsc D.C., Yeh Y.H., Dubuc M, Cartier R, Armour A, Ting M, Sakabe M, Shiroshita-Takeshita A, Comtois P, \& Nattel S. Pulmonary Vein Region Ablation in Experimental Vagal Atrial Fibrillation. Role of pulmonary veins versus autonomic ganglia. Circulation. 2008; 117:470-477. 
Li Y, Li W, Yang B, Han W, Dong D, Xue J, Li B, Yang S, \& Sheng L. (2007). Effects of Cilazapril on atrial electrical, structural and functional remodeling in atrial fibrillation dogs. J Electrocardiol, 40, e1-e6.

Liu X.T, Yang J, Shang F.J, Hong C.M., Guo W.G., Wang B, \& Zheng Q.S. (2009). Silencing GIRK4 expression in human atrial myocytes by adenovirus-delivered small hairpin RNA. Mol Biol Rep,36, 1345-1352.

Oh S, Zhang Y, Bibevski S, Marrouche N.F., Natale A, \& Mazgalev T.N. (2006).Vagal denervation and atrial fibrillation inducibility: Epicardial fat pad ablation does not have long-term effects. Heart Rhythm, 3,701-708.

Pappone C, Santinelli V, Manguso F, Vicedomini G, Gugliotta F, Augello G, Mazzone P, Tortoriello V, Landoni G, Zangrillo A,Lang C, Tomita T, Mesas C, Mastella E, Alfieri O.(2004). Pulmonary vein denervation enhances long-term benefit after circumferential ablation for paroxysmal atrial fibrillation. Circulation, 109,327334.

Platt M, Mandapati R, Scherlag B.J. (2004). Limiting the number and extent of radiofrequency applications to terminate atrial fibrillation and subsequently prevent its inducibility [abstract]. Heart Rhythm, 1, S11.

Sarmast F, Kolli A, Zaitsev A, Parisian K, Dhamoon A.S., Guha P.K., Warren M, Anumonwo J.M., Taffet S.M., Berenfeld O, \& Jalife J. (2003).Cholinergic atrial fibrillation: I KACh gradients determine unequal left/right atrial frequencies and rotor dynamics. Cardiovasc Res, 59,863-873.

Scherlag B.J., \& Po S. (2006). The intrinsic cardiac nervous system and atrial fibrillation. Curr Opin Cardiol, 21, 51-54

Scherlag B.J., Patterson E, \& Po S. (2006). The neural basis of atrial fibrillation. J Cardiovasc Electrophysiol, 39, S180-183.

Sharifov O.F., Fedorov V.V., \& Beloshapko G.G., Glukhov A.V., Yushmanova A.V., \& Rosenshtraukh L.V. (2004). Roles of adrenergic and cholinergic stimulation in spontaneous atrial fibrillation in dogs.J Am Coll Cardiol, 43, 483-490.

Schauerte P, Scherlag B.J., Patterson E, Scherlag M.A., Matsudaria K, Nakagawa H, Lazzara R, \& Jackman W.M. (2001). Focal atrial fibrillation: experimental evidence for a pathophysiologic role of the autonomic nervous system. J Cardiovasc Electrophysiol, 12,600-601.

Tan A.Y., Zhou S, Ogawa M, Song J, Chu M , Li H.M., Fishbein M.C., Lin S.F., Chen L.S., \& Chen P.S.(2008).Neural mechanisms of paroxysmal atrial fibrillation and paroxysmal atrial tachycardia in ambulatory canines. Circulation, 118,916-925.

Tan A.Y., Li H, Wachsmann-Hogiu S, Chen L.S., Chen P.S., Fishbein M.C. (2006). Autonomic innervation and segmental muscular disconnections at the human pulmonary veinatrial junction: implications for catheter ablation of atrial-pulmonary vein junction. J Am Coll Cardiol, 48,132-143.

Wang H, Li J, Hong C, Liu X, Shang F, He Y, Wang Z, \& Zheng Q. (2010) Epicardial ablation of right pulmonary artery ganglionated plexi for the prevention of atrial fibrillation originating in the pulmonary veins. J Electrocardiol, 43, 367-372 
Yang Y.H., Zheng Q.S., Li J, Shang F.J., Liu T, Wang H.T., Liu X.T., \& Liu L. (2009). Agerelated changes in the atrial muscarinic type 2 receptor and their effects on atrial fibrillation vulnerability in rabbits. Experimental Gerontology 44, 572-578. 


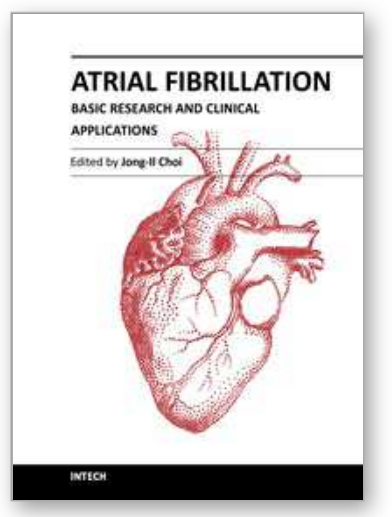

\author{
Atrial Fibrillation - Basic Research and Clinical Applications \\ Edited by Prof. Jong-II Choi
}

ISBN 978-953-307-399-6

Hard cover, 414 pages

Publisher InTech

Published online 11, January, 2012

Published in print edition January, 2012

Atrial Fibrillation-Basic Research and Clinical Applications is designed to provide a comprehensive review and to introduce outstanding and novel researches. This book contains 22 polished chapters and consists of five sections: 1. Basic mechanisms of initiation and maintenance of atrial fibrillation and its pathophysiology, 2. Mapping of atrial fibrillation and novel methods of signal detection. 3. Clinical prognostic predictors of atrial fibrillation and remodeling, 4. Systemic reviews of catheter-based/surgical treatment and novel targets for treatment of atrial fibrillation and 5. Atrial fibrillation in specific conditions and its complications. Each chapter updates the knowledge of atrial fibrillation, providing state-of-the art for not only scientists and clinicians who are interested in electrophysiology, but also general cardiologists.

\title{
How to reference
}

In order to correctly reference this scholarly work, feel free to copy and paste the following:

Hong-Tao Wang, Qiang-Sun Zheng, Xiong-Tao Liu, Jun Li, Fu-Jun Shang, Fei-Fei Su, Bo-yuan Fan and Dong-Bo Ou (2012). Non- Isolation Treatment of Atrial Fibrillation: Does Autonomic Nerve Modulation Really Act?, Atrial Fibrillation - Basic Research and Clinical Applications, Prof. Jong-II Choi (Ed.), ISBN: 978-953-307399-6, InTech, Available from: http://www.intechopen.com/books/atrial-fibrillation-basic-research-and-clinicalapplications/non-isolation-treatment-of-atrial-fibrillation-does-autonomic-nerve-modulation-really-act-

\section{INTECH}

open science | open minds

\author{
InTech Europe \\ University Campus STeP Ri \\ Slavka Krautzeka 83/A \\ 51000 Rijeka, Croatia \\ Phone: +385 (51) 770447 \\ Fax: +385 (51) 686166 \\ www.intechopen.com
}

\author{
InTech China \\ Unit 405, Office Block, Hotel Equatorial Shanghai \\ No.65, Yan An Road (West), Shanghai, 200040, China \\ 中国上海市延安西路65号上海国际贵都大饭店办公楼 405 单元 \\ Phone: +86-21-62489820 \\ Fax: +86-21-62489821
}


(C) 2012 The Author(s). Licensee IntechOpen. This is an open access article distributed under the terms of the Creative Commons Attribution 3.0 License, which permits unrestricted use, distribution, and reproduction in any medium, provided the original work is properly cited. 